\title{
Percutaneous endoscopic gastrostomy feeding in patients with cystic fibrosis
}

\author{
S G J Williams, F Ashworth, A McAlweenie, S Poole, M E Hodson, D Westaby
}

\begin{abstract}
Background-Malnutrition is a common management problem in patients with cystic fibrosis (CF). Various approaches to supplemental nutrition by both parenteral and enteral routes have been used. Aim-To analyse the efficacy and acceptability of supplemental overnight feeding using a percutaneous endoscopic gastrostomy (PEG) in patients with CF.

Patients-53 patients with CF (43 adults; age $>17$ years) with severe pulmonary disease.

Methods-The technical success and complications of PEG insertion were documented together with changes in nutritional and pulmonary status of the cohort.

Results-PEG tubes were successfully inserted in all patients, with immediate complications (respiratory depression) in two $(4 \%)$ and late complications in 13 $(25 \%)$. Feeding was well tolerated by $50 / 51$ $(98 \%)$ of the cohort during a mean (SEM) follow up of 14.5 (2.1) months. The adult cohort had a significant increase in weight and body mass index at six months which was maintained at 12 months. Serum albumin concentration remained stable at six months but had fallen by 12 months, although the differences were not statistically significant. These results were reflected in the paediatric cohort. Pulmonary function in those followed up for one year had apparently stabilised, but the number of admissions to hospital over the year before and the year after PEG did not change. Half of the cohort were accepted for heart-lung/lung transplantation, the improvement in nutritional status being a prerequisite for this.

Conclusion-Supplemental PEG tube feeding is well tolerated and results in a significant improvement in nutritional status and an apparent stabilisation of pulmonary function in severely malnourished CF patients with advanced pulmonary disease.

(Gut 1999;44:87-90)
\end{abstract}

Keywords: cystic fibrosis; malnutrition; enteral nutrition; percutaneous endoscopic gastrostomy

Malnutrition is a common problem in cystic fibrosis (CF). ${ }^{1}$ Its aetiology is probably multifactorial, with proposed mechanisms including the increased resting energy expenditure documented in $\mathrm{CF}^{2}$ deficiency of pancreatic enzymes, ${ }^{3}$ and increased energy requirements associated with chronic pulmonary disease and continuing sepsis. ${ }^{34}$ In addition, chronic illness is associated with anorexia. ${ }^{5}$

Improving nutritional status in patients with CF is important both in terms of improving survival and maintaining pulmonary function. ${ }^{4}$

The approach to improving nutrition in these patients is multidisciplinary and includes appropriate education and dietary advice, optimisation of pancreatic enzyme replacement, and treatment of sepsis. ${ }^{1}$ However, in a proportion of patients, particularly those with more advanced pulmonary disease, these measures alone are insufficient to maintain body weight. ${ }^{1}$

After failure of conservative dietary measures to improve nutritional status, additional interventions have included the use of total parenteral nutrition, ${ }^{57}$ nasogastric feeding, ${ }^{9}{ }^{10}$ and percutaneous endoscopic gastrostomy (PEG) feeding. ${ }^{112}$

PEG feeding has been applied successfully in $\mathrm{CF}$ and has been the preferred method for delivering supplemental nutrition at Brompton Hospital since 1988. The present report assesses the acceptability and effectiveness of PEG feeding in improving nutritional status in $\mathrm{CF}$ patients with severe underlying lung disease.

\section{Patients and methods}

PATIENTS

Royal Brompton Hospital is a level $1 \mathrm{CF}$ centre with its own local catchment of patients, but which also acts as a tertiary referral centre for patients with problems that have proved difficult to manage at their local CF centre and for those referred for consideration for lung transplantation. This results in a heterogeneous patient population.

Patients were selected for PEG insertion on the basis of severe malnutrition (body mass index $(\mathrm{BMI})<17 \mathrm{~kg} / \mathrm{m}^{2}$ ) and a failure to respond to intensive dietary counselling coupled with optimisation of medical status.

\section{METHODS}

Freka gastrostomy tubes (9F; Fresenius, Runcorn, Cheshire, UK) were inserted endoscopically, as previously described, ${ }^{13}$ with intravenous sedation (midazolam/pethidine), supplemental oxygen via nasal cannulae, and antibiotic prophylaxis (cefuroxime $750 \mathrm{mg}$ ) if the patient was not already on broad spectrum antibiotics.

Care of the feeding tube on the ward was carried out using a standardised protocol. Six hours after insertion of the tube, if the patient had been haemodynamically stable and there was no evidence of an ileus, an infusion of sterile water $(50 \mathrm{ml} / \mathrm{h})$ was commenced. The feed
Accepted for publication 22 July 1998 
was started six hours later if the water had been well tolerated.

Supplemental feeding was performed overnight so as not to disrupt the daily activity of the patients and to encourage continued oral nutritional intake during the day. Feeding was with a concentrated modular elemental feed (Elemental O28; Liquigen (SHS, Liverpool, UK) and Polycose (Abbott Laboratories, Maidenhead, Bucks, UK) in pancreatic insufficient patients and with a whole protein feed (Ensure Plus $(1.5 \mathrm{kcal} / \mathrm{ml})$; Abbott Laboratories) in the pancreatic sufficient patients. In 1993, 14 of the pancreatic insufficient patients were changed to a nutritionally complete elemental formula designed specifically for patients with CF (Emsogen; SHS).

The feed was tailored to individual requirements with a maximum provision of $1000 \mathrm{ml}$ and $2.6 \mathrm{kcal} / \mathrm{ml}$, usually providing $40-60 \%$ of the recommended daily energy intake.

Tolerance of the feed was monitored daily immediately after PEG insertion, at 7-10 days after discharge, and subsequently every one to three months depending on the clinical requirements. At each visit the patients were assessed for complications of feeding and PEG placement, nutritional status, and changes in respiratory function.

\section{STATISTICAL ANALYSIS}

The results are expressed as mean (SEM) or median (range) as appropriate. Differences in static variables were assessed using the $\chi^{2}$ test and differences in continuous variables by analysis of variance or the Mann-Whitney U test as appropriate. $\mathrm{p}<0.05$ was considered statistically significant for a two tailed test.

For analysis of the nutritional data, the cohort was divided on the basis of age to discriminate between paediatric $(\leqslant 17$ years old) and adult ( $>17$ years old) subjects.

\section{Results}

Over the six years from March 1988 to December 1994, 53 patients with CF were considered to be in need of supplemental feeding after the failure of conservative measures. Thirty nine were female, with a mean (SEM) age of $22.0(0.8)$ years. Forty three of the cohort fell into the adult subgroup (33 women, 10 men; mean age 23.7 (0.8) years) and 10 into the paediatric subgroup (six girls, four boys; mean age 14.7 (0.7) years). Fifty were pancreatic insufficient, the diagnosis being based on symptoms suggestive of steatorrhoea, three day faecal fat estimation, and response to pancreatic enzyme supplements.

PEG tubes were successfully inserted into all 53 patients. Immediate complications occurred in two (4\%), who experienced respiratory depression necessitating reversal of sedation with flumazenil/naloxone and in one case overnight support with nasal continuous positive airway pressure. Two patients (4\%), early in the series, died within one week of PEG insertion. These deaths were the consequence of preexisting severe lung disease; there was no evidence in either case that the deaths were related to the insertion of the PEG tube.
Table 1 Changes in weight, BMI, and serum albumin for the adult subgroup after percutaneous endoscopic gastrostomy feeding. Data presented are those for subjects with 12 month follow up data

\begin{tabular}{llll}
\hline & Before & 6 months & 12 months \\
\hline Weight $(\mathrm{kg})(\mathrm{n}=22)$ & $38.1(0.9)$ & $44.5(1.2)$ & $44.2(1.3)$ \\
BMI $\left(\mathrm{kg} / \mathrm{m}^{2}\right)(\mathrm{n}=21)$ & $15.0(0.3)$ & $17.7(0.5)$ & $17.7(0.4)$ \\
Albumin $(\mathrm{g} / \mathrm{l})(\mathrm{n}=23)$ & $33.8(1.4)$ & $34.6(1.4)$ & $30.9(1.8)$ \\
\hline
\end{tabular}

Results are expressed as mean (SEM).

Late complications were observed in $13 / 51$ $(25 \%)$ of the patients. In two $(4 \%)$ these could be considered serious, with one experiencing unexplained pain in association with PEG feeding, which necessitated PEG tube removal, and the other having the tube removed after displacement. In a further 10 patients the problem was related to local pain or low grade sepsis, which responded to analgesics and/or appropriate antibiotics. In one case the feeding tube became occluded, requiring the tube to be changed. Six patients had their PEG tube changed electively.

PEG feeding was well tolerated by $50 / 51$ (98\%) of the patients during a mean follow up of 14.5 (2.1) months. The mean supplemental energy delivered through the PEG tube was 1490.7 (49.8) kcal.

During follow up, 25 of the whole cohort were accepted on to the heart-lung/lung transplant list. A prerequisite for transplantation was a significant improvement in nutritional status. Four patients successfully underwent transplantation, although only two are still alive. Sixteen died during the initial 12 month follow up period, with seven patients dying within six months of PEG insertion and a further nine dying within 12 months.

During follow up there was a statistically significant increase in weight and BMI of both adult and paediatric subgroups. In the adult subgroup, weight increased from $37.4(0.8) \mathrm{kg}$ before feeding to 42.1 (1.1) $\mathrm{kg}$ at six months ( $\mathrm{n}=37 ; \mathrm{p}=0.0001)$. This improvement was maintained at 12 months in the 22 subjects for whom data were available $(p=0.0001)$ (table 1).

BMI increased from 14.9 (0.4) to $17.7(0.5)$ $\mathrm{kg} / \mathrm{m}^{2}$ at six months $(\mathrm{n}=25 ; \mathrm{p}=0.0001)$. Again the improvement was maintained at 12 months (table 1).

Serum albumin $(\mathrm{g} / \mathrm{l})$ remained stable $(32.8$ (1.3) to $34.2(1.5) ; \mathrm{n}=26)$ at six months, but fell slightly in those patients for whom follow up data were available at 12 months, although the differences were not statistically significant (table 1).

In the paediatric subgroup, weight increased from 31.9 (2.7) $\mathrm{kg}$ before feeding to 35.3 (3.3) $\mathrm{kg}$ at six months $(\mathrm{n}=9 ; \mathrm{p}<0.02)$. This improvement was also maintained at 12 months in the six subjects for whom data were available $(\mathrm{p}<0.02)$ (table 2). Serum albumin was maintained over 12 months in those subjects for whom follow up data were available (table 2).

The predicted lung function parameters of the whole cohort did not change significantly during follow up. Thus the percentage predicted forced expiratory volume in one second 
Table 2 Changes in weight and serum albumin for the paediatric subgroup after percutaneous endoscopic gastrostomy feeding. Data presented are those for patients with 12 month follow up data

\begin{tabular}{llll}
\hline & Before & 6 months & 12 months \\
\hline Weight $(\mathrm{kg})(\mathrm{n}=6)$ & $30.3(3.8)$ & $34.8(4.8)$ & $35.1(4.7)$ \\
Albumin $(\mathrm{g} / \mathrm{l})(\mathrm{n}=5)$ & $36.0(1.9)$ & $37.0(0.9)$ & $34.8(1.5)$
\end{tabular}

Results are expressed as mean (SEM).

$\left(\mathrm{FEV}_{1}\right)$ was $21(13-35) \%$ before PEG tube insertion and $20(13-35) \%$ at six months, and the percentage predicted forced vital capacity (FVC) was 38 (19-66)\% before PEG and 37 $(17-84) \%$ at six months. Respiratory function remained stable in the group for whom follow up data were available at 12 months (table 3 ).

Despite the improved nutritional status of the whole cohort, there was no reduction in the number of admissions to hospital for acute respiratory exacerbations during the year after PEG insertion (median (range) four (zero to six) admissions) when this was compared with the 12 months preceding PEG insertion (three (one to seven) admissions).

\section{Discussion}

Improving the nutritional status of patients with $\mathrm{CF}$ is extremely important in their long term management. It has been suggested that the early restoration of nutritional status may result in improved response to treatment for respiratory exacerbations, amelioration of the progressive decline in lung function, extended survival, ${ }^{46}$ and perhaps improved quality of life. In addition, it is a prerequisite of acceptance for lung transplantation that the nutritional status of the patient has been optimised.

After the failure of conventional dietary therapy, optimisation of pancreatic replacement therapy, and use of high energy oral supplements, the approach to supplemental nutrition has been mainly through the enteral and occasionally the parenteral routes.

The parenteral route has been used successfully in the short term, ${ }^{58}$ with some workers reporting weight gain and an improvement in respiratory function over a six month time frame (after a three week feeding period), but only in a small cohort of patients. ${ }^{5}$ However, there is evidence that the benefits are lost soon after feeding is discontinued. ${ }^{14}$ Other disadvantages of this approach include its expense and the complex arrangements and monitoring requirements in the home setting. It also carries a significant risk of complications such as sepsis and thrombosis.

Supplemental enteral feeding generally involves the delivery of an overnight feed by the nasogastric, gastrostomy, or jejunostomy route.

Table 3 Lung function data for the whole cohort before and after percutaneous endoscopic gastrostomy feeding. Data presented are those for patients with 12 month follow up data

\begin{tabular}{llll}
\hline & Before & 6 months & 12 months \\
\hline$\%$ predicted FEV $(\mathrm{n}=15)$ & $22(14-30)$ & $21(13-35)$ & $22(10-40)$ \\
$\%$ predicted FVC $(\mathrm{n}=15)$ & $33(20-46.5)$ & $37(23.6-54)$ & $36(18-60)$ \\
\hline
\end{tabular}

Results are expressed as median (range)

$\mathrm{FEV}_{1}$, forced expiratory volume in one second.

FVC, forced vital capacity.
Although results using the nasogastric route have been encouraging, ${ }^{9}{ }^{10}$ the potential problems of inserting the tube, particularly in a patient with nasal polyps (a common problem in adults with $\mathrm{CF}$ ), the need to repeatedly insert the tube which may result in nasal irritation, and the potential for tube displacement (particularly in patients who have a chronic cough) resulting in the possibility of aspiration of the feed and potential loss of valuable supplemental feeding time make this route less acceptable. $^{1015}$ Additional complications of nasogastric feeding include nasopharyngeal sepsis, oesophageal erosion, and inhibition of oral feeding, ${ }^{15}$ together with an adverse effect on the morale of both patient and family. ${ }^{16}$

The ease of insertion of gastrostomy tubes using the endoscopic pull technique is such that many now consider this to be the method of choice for achieving long term enteral nutritional intervention. This approach has been shown to produce superior results in terms of nutritional benefit when compared with nasogastric feeding. ${ }^{15} 17$ Our results confirm the practicalities of inserting PEG tubes under intravenous sedation with careful monitoring in $\mathrm{CF}$ patients with severe chronic respiratory insufficiency. The necessity of monitoring patients carefully is emphasised by the two cases of respiratory depression. The two deaths in this series occurred early on in the experience of PEG insertion in this group of patients. Both patients had end stage lung disease with gross malnutrition and would not now be considered suitable candidates for PEG insertion. The results also show that the use of the PEG is well tolerated, even in a group of severely debilitated patients, with few significant complications related to the tube itself.

The improvement in nutritional status, as assessed by gains in weight and BMI, in a group of patients who would be considered severely malnourished (BMI <17) is highly significant, showing the efficacy of this method of supplemental feeding. This improvement appeared to be sustained in those patients followed up for 12 months.

The changes in serum albumin are more difficult to interpret, perhaps reflecting the multitude of potential influences on serum albumin concentration. However, the concentration was well maintained at six months, and, although there was a fall at 12 months, this was not statistically significant. Further interpretation of these data would require a control group not receiving supplemental nutrition.

The fact that pulmonary function, in those patients who survived, did not continue to show the expected gradual decline $(2-3 \%$ per annum $)^{18}$ is encouraging, although a larger cohort and longer follow up would be required to comment on this further. This apparent improvement/stabilisation of respiratory status was not supported by the data on hospital admissions for acute infective respiratory exacerbations in the cohort of patients surviving to one year after PEG tube insertion.

PEG feeding in this study was used for a particularly sick cohort of patients. This is reflected by the fact that $25 / 53(47 \%)$ of the 
cohort were accepted for lung transplantation. In the majority, supplemental feeding achieved the necessary improvement in their nutritional status for them to be included on the transplant register.

Whether PEG feeding would have influenced the survival of these patients has not been addressed by this study. This would require a larger cohort with longer follow up. However, it is likely that supplemental feeding provided the bridge to transplantation for the four patients who did undergo heart-lung/lung transplantation. Given the gross malnutrition of many of the cohort, a factor that was to some extent governed by their clinical status on arrival from their referring centre, earlier intervention with supplemental enteral nutrition may lead to improved results. This, however, requires prospective evaluation.

In summary, this retrospective analysis shows that PEG tubes proved simple to insert, with subsequent supplemental feeding using an elemental formula being well tolerated and leading to significant weight gain and apparent stabilisation of pulmonary function in a group of severely malnourished CF patients with advanced pulmonary disease. It allowed half of the cohort to be accepted on to the lung transplant register. Earlier intervention with PEG feeding, before patients become severely malnourished, may lead to improved results. In addition, formal assessment of quality of life may give further insight into the acceptability of this mode of feeding to the patients themselves.

This paper was presented at the 20th European Cystic Fibrosis Meeting, Brussels (1995; abstract O6).

1 Durie PR, Pencharz PB. Nutrition. Br Med Bull 1992;48:823-47.
2 Buchdahl RM, Cox M, Fulleylove C, et al. Increased resting energy expenditure in cystic fibrosis. F Appl Physiol 988;64:1810-16.

3 Lapey A, Kattwinkel J, di Sant'Agnese PA, et al. Steatorrhoea and azotorrhoea and their relation to growth and nutrition in adolescents and young adults with cystic fibrosis. F Pediatr 1974;84:328-34.

4 Kraemer R, Rudeberg A, Hadorn B, et al. Relative underweight in cystic fibrosis and its prognostic value. Acta Paediatr Scand 1978;67:33-7.

5 Shepherd R, Cooksley WGE, Domville Cooke WD. Improved growth and clinical, nutritional, and respiratory changes in response to nutritional therapy in cystic fibrosis. f Pediatr 1980;97:351-7.

6 Sproul A, Huang N. Growth patterns in children with cystic fibrosis. F Pediatr 1964;65:664-76.

7 Lester LA, Rothberg RM, Dawson G, et al. Supplemental parenteral nutrition in cystic fibrosis. 7 Parenteral Enteral Nutr 1986;10:289-95.

8 Skeie B, Askanazi J, Rothkopf MM, et al. Improved exercise tolerance with long term parenteral nutrition in cystic fibrosis. Crit Care Med 1987;15:960-2.

9 Moore MC, Greene HL, Donald WD, et al. Enteral tube feeding as adjunct therapy in malnourished patients with cystci fibrosis: a clinical study and literature review. $A m \mathcal{F}$ Clin Nutr 1986;44:33-41.

10 Pencharz P, Hill R, Archibald E, et al. Energy needs and nutritional rehabilitation in undernourished adolescents and young adult patients with cystic fibrosis. 7 Pediatr Gastroenterol Nutr 1984;3(suppl 1):S147-53.

11 Levy LD, Durie PR, Pencharz PB, et al. Effects of longterm nutritional rehabilitation on body composition and clinical status in malnourished children and adolescents with cystic fibrosis. F Pediatr 1985;107:225-30.

12 Steinkamp G, von der Hardt H. Improvement of nutritional status and lung function after long-term nocturnal gastrostomy feedings in cystic fibrosis. F Pediatr 1994;124:244-9.

13 Russell TR, Brotman M, Norris F. Percutaneous gastrostomy. A new simplified and cost-effective technique. $A m \mathcal{F}$ Surg 1984;148:132-7.

14 Mansell AL, Andersen JC, Muttart CR, et al. Short term pulmonary effects of total parenteral nutrition in children with cystic fibrosis. F Pediatr 1984;104:700-5.

15 Wicks C, Gimson A, Vlavianos P, et al. Assessment of the percutaneous endoscopic gastrostomy feeding tube as part of an integrated approach to enteral feeding. Gut 1992;33: 613-16.

16 Biggart M, McQuillan PJ, Choudhury AK, et al. Dangers of placement of narrow bore nasogastric feeding tubes. Ann $R$ Coll Surg Engl 1987;69:119-21.

17 Park RHR, Allison MC, Lang J, et al. Randomized comparison of percutaneous endoscopic gastrostomy and nasogastric tube feeding in patients with persisting neurological dysphagia. BMF 1992;304:1406-9.

18 Corey M, Levison H, Crozier D. Five- to seven-year course of pulmonary function in cystic fibrosis. Am Rev Respir Dis 1976;114:1085-92. 